\title{
L'anniversario della Grande guerra in Italia. Spunti e contrappunti a metà del guado
}

\author{
Mario Isnenghi
}

"Non siamo qui per celebrare...". Sono i preliminari d'uso, gli scongiuri propiziatori a ogni inizio d'incontro promosso nel Centenario da questo o quell'ente ${ }^{1}$.

E per capire come è andata, no?

Mica tanto. Ricostruire storicamente è un optional. Tanto la storia è finita, la politica è uno schifo, le ideologie sono morte e noi siamo per la pace.

Richiamare all'accaduto suona vecchio barbogio e mette le pastoie al libero effondersi dei bisogni e dei desideri dell'io. Oralità come prosecuzione di Facebook con altro mezzo. Perché mai quel mezzo guerrafondaio sul palco deve venirci a raccontare che qualcuno quella guerra la voleva fare? Io dico di no. E anch'io. 57, 96, 112 dicono di no.

Non siamo ancora a questo, de visu, ma la democrazia bagolona e sbracata di Internet sembra additare il cammino.

La Costituzione ripudia la guerra ${ }^{2}$, lo sappiamo. Ma - per quel che valgono questi richiami - lo Statuto no, ed era la costituzione loro, che si portava dentro i poteri del re e un barlume dei tempi in cui era nato, l'epica del Quarantotto.

"Dovevi occuparti di Caporetto per tutta la vita", intuisco che mi vogliono far capire alcuni. "Avevi cominciato così bene". "Cos'è questa storia di Vittorio Veneto e che l'Italia ha vinto"? Certe cose non si dicono.

Il fatto è che io in quegli anni sessanta ero andato a vedere se i "vinti di Caporetto" stavano facendo la rivoluzione ${ }^{3}$. Era invece qualche cosa di diverso e, secondo me, molto meno: provai a chiamarla "rivolta abortita" per difendermi dalle rampogne di "Rinascita", il settimanale del Pci, e da un Aurelio Lepre allora allineato a sinistra. Era troppo anche quello, mi influenzava Malaparte ${ }^{4}$, con "speranzielle" alla luce dei fatti infondate. In realtà, come si è venuto chia-

${ }^{1}$ Per una panoramica si veda http://www.centenario1914-1918.it/it (ultimo accesso 14 gennaio 2016).

${ }^{2} \mathrm{Si}$ vedano Guido Neppi Modona (a cura di), Stato della Costituzione, Milano, il Saggiatore, 19982; Nicola Labanca, Le forze armate e lo Stato democratico, in Maurizio Ridolfi (a cura di), Almanacco della Repubblica. Storia d'Italia attraverso le tradizioni, le istituzioni e le simbologie repubblicane, Milano, Bruno Mondadori, 2003, pp. 299-313.

${ }^{3} \mathrm{Si}$ vedano Mario Isnenghi, I vinti di Caporetto nella letteratura di guerra, Padova, Marsilio, 1967, e Id., Il mito della grande guerra da Marinetti a Malaparte, Bari, Laterza, 1970. Cfr. anche Mario Isnenghi, Passati remoti 1914-1919. Due saggi sulla Grande guerra, Roma, Edizioni dell'asino, 2014.

${ }^{4} \mathrm{Si}$ veda Curt Erich Suchert (Curzio Malaparte), Viva Caporetto, Prato, Stab. lito-tipografico M. Martini, 1921. Si veda anche Curzio Malaparte, Viva Caporetto! La rivolta dei santi maledetti, introduzione di Mario Isnenghi, Milano, Mondadori, 1980. 
rendo, sulla sconfitta militare si è sovrapposto, ha cominciato a serpeggiare un "tutti a casa", che poteva essere un grido di liberazione o di orrore, comunque disgregativo. Se poi il processo disgregativo si ferma, i soldati italiani non scappano da tutte le parti, l'avanzata si arresta, l'esercito tiene, sei mesi dopo, nella battaglia di giugno, continua a tenere e, a questo punto, il processo di crisi si inverte e investe ormai il blocco avversario, si arriva all'ottobre-novembre 1918, e adesso sono "loro" che si disgregano: cos'è, "colpa mia"? O è invece qualche cosa da comprendere, un processo da ricostruire, un lessico e dei sensi di sé da adeguare all'accaduto?

C'erano già allora, del resto, attese e interpretazioni diverse. In un altro libro periodizzante della fase uscito appena un anno dopo il mio, in coincidenza con il Sessantotto, la parte innovativa erano le sentenze dei tribunali militari con quella sberla di titolo - Plotone d'esecuzione - piantato al centro in bella vista ${ }^{5}$; era spiazzante anche l'Elogio della paura, l'introduzione con cui Enzo Forcella si affiancava al lavoro dello storico e dirigente dell'Azione cattolica Alberto Monticone; e, anticipando i tempi, confessava implicitamente quel che più avanti dirà a piena voce, di aver voluto nel 1943-1944 rifugiarsi in Vaticano e tentare di fare la Guardia svizzera, tutt'altro che la resistenza armata: che non sarà confessione da poco per chi, nel contempo, dirigeva un Istituto di storia della Resistenza. Ma additare i motivi e le umane virtù della fuga dalla violenza e dalla guerra appariva allora dissonante. Io certo non mi ci ritrovavo e non mancai di dirlo pubblicamente in una presentazione del loro libro in una libreria di Mestre, che ancora mi mette a disagio perché Forcella non se l'aspettava e, come presentazione, era davvero scostumata.

Descrizione sintetica di una parabola mentale collettiva: dalla vittoria mutilata - già grottesca e masochista di suo - alla guerra mutilata della vittoria. A ogni stagione il suo "politicamente corretto". 4 Novembre: omissis. Rifiutarsi di ammettere questa cosa sconcertante, di aver vinto: potremmo considerarla il capolavoro di una "impetuosa propaganda di autodisprezzo" nello "sforzo d'introspezione autoingiuriosa", facendo nostre espressioni dello scrittore ed esule antifascista G.A. Borgese ${ }^{6}$ : che è poi la ben nota struttura permanente dell' $A n$ titaliano', alimentata da destra e da sinistra, e oggi reinvestita e resa virtuosa con gli assoluti della Pace.

Sono partito dai Vinti e da quella rivoluzione che non c'era: e lì però ho imparato a viaggiare sempre su un doppio binario, gli immaginari e i fatti, ovvero i fatti di primo e di secondo grado, compresi i "fattoidi". Ho battuto e ribattuto su questo: io sono più portato a lavorare sugli immaginari - mi viene dalle mie matrici letterarie - ma ho imparato presto e me la sono tenuta per detta,

\footnotetext{
${ }^{5}$ Si veda Enzo Forcella, Alberto Monticone, Plotone di esecuzione. I processi della Prima guerra mondiale, Bari, Laterza, 1968.

${ }^{6} \mathrm{Si}$ veda Giuseppe Antonio Borgese, Goliath. The March of Fascism, New York, The Viking press, 1937 (prima ed. ital. Golia. Marcia del fascismo, Milano, Mondadori, 1946).
} 
quella reciproca complicazione di fattuale e virtuale o percepito. Non bastano i fantasmi, le ipotesi, il verosimile, il creduto vero, il desiderio: ci vogliono gli accertamenti fattuali. Ma ci vogliono tutti e due, nessuna rinuncia e nessun superamento, non mi gioco uno contro o al posto dell'altro. Ho imparato allora e ho continuato così, non è che il centenario sia fatto per smentirmi, anzi. Magari, invece che l'ombra di Lenin trapela quella di papa Benedetto e al mito della rivoluzione subentra e si diffonde quello dell'"inutile strage": un "anti-Stato" surrogatorio.

Richiamo ai fatti avvenuti quando, sempre più spesso - nell'anniversario in corso del 1914-1918, come già nel 2011 in quello del 1861 - mi sembra che si inclini a prescinderne; inalbero scherzando la bandiera di un nuovo positivismo; e però non sta a me rinchiudermi nei "fatti in sé", lasciando agli altri gli spazi della ricezione, delle interpretazioni e dell'immaginario. Lo studioso dei luoghi della memoria sono io ${ }^{7}$, non rinuncio alla memoria perché mi trovo a dover contrastare chi la confonde con la storia. Sarà anche per questo che mi piace - mi sembra dia una marcia in più, a tutti e non solo a chi parla quando il discorso pubblico trova la sua collocazione in un teatro che sia esso stesso una infrastruttura della storia collettiva. E così, appunto, sei lì a parlare di storia, ma dentro un luogo della memoria, un contenitore impregnato di vita collettiva, della storia di più generazioni, e forse in parte questa autocoscienza si riverbera negli ascoltatori, cui spetta ora di sedere in quella platea e in quei palchi: alcuni sanno, i più possono forse intuire in qualche modo lo spirito dei luoghi, per più intense connessioni con il succedersi dei tempi. È un "evento", non solo nel senso banalizzante degli addetti alla comunicazione. Sarà questa la ragione per cui se l'editore Laterza e altri ambientano i loro cicli di incontri in luoghi come questi si mettono in fila per esserci mille persone, mentre quella "stessa" conferenza, in una delle solite sale, ne raccoglierebbe qualche decina? A me è avvenuto di parlare delle Ore di Trieste al Teatro Verdi di Trieste, del 1914-'15 come Capolinea di miti al Teatro Sociale di Trento ${ }^{8}$ : il mio personale rientrare nel viluppo di circostanze e affabulazioni del "mito di Trentoe-Trieste". Non era lo stesso che pronunciare o ascoltare le stesse parole in un luogo neutro.

A costo di farmi sgridare perché rivaluterei l'interventismo democratico, ammetto di essere salito per prima cosa al Doss Trento la sera prima dell'esibizione al "Sociale". Quanti decenni erano che non salivo al monumento a Battisti? Sarà eroicizzante, ma se la guerra è, come è, tragedia, niente la riassume me-

${ }^{7} \mathrm{Si}$ veda Mario Isnenghi (a cura di), Luoghi della memoria. Simboli e miti dell'Italia unita, 3 voll., Roma-Bari, Laterza, 1996-1997.

${ }^{8}$ Mario Isnenghi, conferenza su Trento e la Grande guerra, nel ciclo Storia di Trieste, introdotto da Claudio Ernè, Trieste, Teatro Verdi, 14 dicembre 2013; conferenza su Epoche. Capolinea dei miti, nel ciclo L'Italia in guerra, introdotto da Camillo Zadra, Trento, Teatro sociale, 18 ottobre 2015. 
glio di quella personalità e di quella fine. Sera di ottobre, quasi buio, le grandi colonne si stagliano ancora, ma sotto, nella cripta, è già buio. Si distinguono però le parole nel registro dei visitatori, cui mi avvicino per leggere - lo faccio sempre, è una buona fonte - temendo di scoprire quello che credo di antivedere: che saranno pochi e, quei pochi, antipatizzanti e ostili. Non è così. Vedo, dalle frasi e dalle firme, un'affluenza continuata, da varie parti d'Italia e anche da fuori, direi che siano meno quelle locali. Le dichiarazioni di ostilità ci sono - più in nome dei valori del Tirolo che dei valori della pace - ma meno numerose del prevedibile in base alle contumelie, terrificanti, di cui è gratificato Battisti nei siti internet degli "Austriaci d'Italia", come ho visto che qualcuno, per contrappasso, si compiace di denominarsi. Quanto alla maggioranza, sembra non saper bene che linguaggio usare: è ammirativa, dichiara gratitudine, con un che di tradizionale e di vetusto, quasi non si riuscisse ad aggiornare e dar forma a una scolastica datata.

Fino a tempi non poi così remoti - diciamo anni novanta inoltrati - sono sicuro che Marina Rossi mi avrebbe ricompreso, e forse anche messo in testa all'elenco degli amici e compagni di strada che sente affini e compartecipi della sua ricerca sulla Grande guerra e che nomina in apertura del suo recente 1915: l'Italia in guerra. Esperienza e memorie degli italiani delle ex Terre Irredente ${ }^{10}$.

Ora, non più. Cos'è accaduto? Sembra come se si siano venuti formando, in tema di Grande guerra, due "partiti" storiografici. Io però so di essere trasversale e rilutto a lasciarmi murare in non so quale altro partito rispetto a quello adombrato dal suo elenco, e i cui singoli componenti - che quindi evito qui di specificare - non è poi detto che si riconoscano nell'identità cui parrebbero assegnati in blocco. Chi sono, gli adepti di quello che qualcuno ha chiamato "paradigma vittimario"?

Sovversivi ed ex sovversivi di metà Ottocento gravitano verso lo Stato-guida, che è allora il Piemonte. Sovversivi ed ex-sovversivi del 1914-15 gravitano verso lo Stato-guida, che ora è il regno d'Italia o, all'estero, gli altri Paesi e Stati, con l'eccezione della Russia. È andata così. Con il capo dei riformisti nel partito socialista, Turati, che invoca Giolitti, il demiurgo, e nell'intimità, con Anna Kuliscioff, lo chiama "Giovannino" solini, che abiura la pace, molla il partito, grida "viva la guerra" e fonda movimenti e giornale per la guerra. Davvero non c'era e non c'è nulla da problematizzare e da capire e ci si può limitare ad asserragliarsi in presupposti pietosi e

${ }^{9} \mathrm{Si}$ veda http://www.austriaciditalia.it/, http://www.austriaciditalia.it/blog.html e https://austriaciditalia.wordpress.com/.

${ }^{10} \mathrm{Si}$ veda Marina Rossi, 1915 l'Italia in guerra. Esperienza e memorie degli italiani delle exTerre irredente, Treviso, ES, 2015.

${ }^{11} \mathrm{Si}$ veda Filippo Turati, Anna Kuliscioff, Carteggio, 6 voll., raccolto da Alessandro Schiavi, a cura di Franco Pedone, Torino, Einaudi, 1949-1959 (ried. 1977) (il volume sulla Grande guerra è il terzo). 
non-violenti "senza se e senza ma"? A ciascuno, unilateralmente, la sua quota di vittime della guerra, e guai a chi si provi a guardare il paesaggio storico nel suo insieme e a cercare di vedere se e come convivano i "sł" e i "no", visto che i "no" e i "si”" ci sono, e anche internamente differenziati...

A Bologna, di recente, presentando una ristampa del libro del 2000 di Rochat e mio su La Grande guerra 1914-1918 ${ }^{12}$ Giovanna Procacci dice ${ }^{13}$ che la nostra è ormai la "storiografia del consenso"; e che ci siamo sdraiati sulla "classe dirigente". Aveva preannunciato che sarebbe stata una "provocazione", ma era difficile aspettarsi tanto. Stando al gioco, elenco i Salandra, i Sonnino, i Boselli, gli Orlando dietro cui dovrei allinearmi, mostrandone a smorfie l'improbabilità; finché mi lascio sfuggire che la figura più rispettabile e grave, quella all'altezza della tragedia e che mi fa pensare, sarebbe stata Battisti. E se nel dopoguerra è diventato presidente del consiglio Bonomi... Ma forse è un'ammissione di colpa.

Già nel 2000, alla sua prima uscita, quel nostro libro - che passa peraltro di ristampa in ristampa - fu oggetto di rampogne deluse, in sedi e in particolare da parte di un recensore imprevedibile. Laria andava cambiando. E non solo per la nota stonata del nostro non associarsi al paradigma vittimario, ma anche per quella pretesa di fare storia complessiva nell'ora del relativo, o dell'assolutizzazione dei relativi.

Il treno passa di fiume in fiume penetrando nei luoghi della guerra. Sto andando a Gorizia, a un Liceo "Dante". Ho riguardato gli appunti che ho preso, stavolta è una panoramica più vasta, venticinque anni, giusto una generazione: Da Vamba a Omodeo: autonarrazioni e racconti ${ }^{14}$. Ora passo ai giornali. Ed ecco sul "Manifesto", un clic, come avrebbe detto Leo Spitzer; e questo spicilegio è fatto di clic: notizie di viaggio da dentro l'anniversario. A fondo pagina 7 (26 novembre 2015) vedo la pubblicità di uno "spettacolo-concerto" sulla Grande guerra, promosso da sigle che mi sono care, come il Circolo "Gianni Bosio", e vedo il nome di Gramsci. Titolo: Maledetti studenti italiani /che la guerra l'avete voluta. Vero. Molti di loro. Animavano le mobilitazioni per l'intervento. È un fatto.

E ora il sottotitolo: Cento anni fa la Prima guerra mondiale / costò al nostro popolo 1.200.000 morti $^{15}$. Il "nostro popolo"? è già una notizia. Non sarà una bieca caduta nel "nazionalismo"? Ma poi: 1.200 .000 morti? La cifra effettiva è rimasta incerta, ma oscilla intorno ai 600.000, la metà (a meno di non

${ }^{12} \mathrm{Si}$ veda Mario Isnenghi, Giorgio Rochat, La grande guerra 1914-1918, Firenze, La Nuova Italia, 2000, varie ried. Bologna, Il Mulino, 2015 la più recente.

${ }^{13}$ Giovanna Procacci, presentazione del volume di Mario Isnenghi e Giorgio Rochat, La Grande guerra 1914-1918, Bologna, Istituto Parri, 12 novembre 2015. Presentavano anche Fiorenza Tarozzi e Andrea Battistini.

${ }^{14}$ Mario Isnenghi, conferenza su Da Vamba a Omodeo: autonarrazioni e racconti, Gorizia, Liceo classico "Dante Alighieri", 26 novembre 2015.

15 "Il manifesto", 26 novembre 2015. 
contare anche i morti per malattia: la "spagnola"). Questo, dunque, non è un fatto, ma semplicemente una falsificazione. Ma senza andare a sindacare questa contabilità comunque sinistra, si può però constatare il disinvolto maneggio delle cifre. Prioritario sembra essere emozionare e scandalizzare, non informare e argomentare. Questi canti della guerra, magari postumi, li pratico e li canto anch'io, da quel dì. Ma non mi bastano. E ricordo anche quali — proprio i più belli — c'erano indizi che fossero nati cinquant'anni dopo, così, per sollecitare la vena popolare. Le politiche della memoria variano, ma ci sono in ogni tempo.

"Noi Wu Ming diciamo addio alla Storia"16. Bene così. Lasciatela agli storici.

La Rete delle Geostorie a scala locale mi fa avere delle domande che mi pare possano rappresentare la difformità di attese e di linguaggi che questo centenario porta con sé: con le responsabilità e gli accenti di chi fa scuola, come il professor Ernesto Perillo che mi contatta a nome di un gruppo organizzato di colleghi didatticamente impegnati ${ }^{17}$.

Le domande:

1. La guerra. O meglio le guerre. Per limitarci a quelle degli italiani degli ultimi due secoli a cui lei ha dedicato molti dei suoi studi, che cosa ci insegnano della (nostra) storia?

2. Se e come è cambiata la guerra, il modo di farla, pensarla, raccontarla nel corso del tempo?

3. E la pace?

4. Conoscere e insegnare le guerre può servire a conoscere e imparare la pace? In che modo? A quali condizioni?

5. Per il racconto storico la guerra sembra essere un protagonista assolutamente principale sulle cui sorti si costruisce gran parte dell'intreccio della storia. E la pace resta in fondo una conseguenza (in qualche modo inevitabile e un po' ovvia) della cessazione della guerra, in attesa del prossimo conflitto, senza mai assumere un ruolo autonomo e degno di essere raccontato di per sé. Quali le ragioni di questa asimmetria?

6. Anche gli studenti spesso sono affascinati dalla guerra come tema privilegiato del racconto storico. Se e come gli insegnanti possono "smontare" il fascino che la guerra continua ad avere presso i ragazzi?

7. La gran parte dei docenti della nostra Rete lavora con alunni della scuola primaria e quando li osserviamo vediamo che interpretano la guerra come un gioco da farsi in cortile, alla playstation, al Pc o da guardare in certi programmi per bambini. Ma se qualcuno di loro la sente raccontare dai nonni o dai bisnonni inizia ad averne una visione un poco diversa. È possibile e giusto parlare della guerra ai bambini di oggi? Partendo da che cosa? Da quali aspetti storici essendo (riteniamo) improponibile parlare di motivazioni politico-economiche? In generale, gli aspetti politici ed economici sono sufficienti per comprendere la guerra? Su quali altri ambiti si può far leva per costruire una conoscenza sensata della guerra?

8. Avrebbe qualche titolo da proporci, tra le sue pubblicazioni, che potrebbe essere utile per progettare percorsi didattici per alunni anche di scuola primaria?

${ }^{16}$ Intervista di Michele Smargiassi, "la Repubblica", 27 novembre 2015, p. 49. Si veda Wu Ming 1, Cent'anni a Nordest. Viaggio tra i fantasmi della guera granda, Milano, Rizzoli, 2015; Wu Ming, L'invisibile ovunque, Torino, Einaudi, 2015; e Wu Ming, L'armata dei sonnambuli, Torino, Einaudi, 2014.

${ }^{17}$ Rete delle GeoStorie a scala locale, "Newletter", n. 8, novembre 2015. 
9. Quanto pesano nel racconto storico della guerra i meccanismi narrativi legati a modelli convenzionali o stereotipi quali la lotta del bene contro il male, l'attribuzione del primato, la conquista del potere, l'esaltazione dell'eroe?

10. Nel racconto storico della guerra è riconoscibile la presenza o l'assenza di elementi legati alla diversità di sistemi valoriali maschili/femminili? In generale, la diversità di genere può essere utile per costruire una visione più corretta della guerra e della pace?

11. La guerra, come ogni altro evento del passato, non è solo ciò che è accaduto, ma anche la memoria nel presente che di quel passato vogliamo ricordare e il modo/ i modi per dirlo. Con riferimento a due recenti anniversari - il centenario della Prima guerra mondiale e il settantesimo della Liberazione - quali sono le caratteristiche della memoria contemporanea di quegli eventi e come a scuola costruire una alfabetizzazione sensata di quel nostro passato?

12. Le guerre sono state e sono dispositivi fondamentali per la nazionalizzazione delle masse e la costruzione di identità collettive e nazionali. Anche nella storia insegnata. È corretto individuare le nazioni quali protagonisti dei due conflitti mondiali? La realtà di un mondo sempre più globale e di un'Europa che cerca di costruirsi come nuovo soggetto politico e culturale se e come modificano il significato di questa prospettiva? Quali soggetti si possono individuare come protagonisti dei conflitti contemporanei?

13. Insegnare e imparare la pace anche attraverso la storia. Una grande illusione? Che cosa dobbiamo insegnare, allora, per costruire la pace?

\section{Se queste erano le domande, ecco qui di seguito le risposte di Mario Isnenghi:}

Mi sono aggirato, con ammirazione e rispetto per il vostro grande lavoro, in internet. E vorrei aiutarvi. Però non così: la natura delle domande non mi aiuta. Certo, sono bellissime domande, le domande "ultime", se vogliamo, in tema di pace e guerra. Però, agli occhi dello storico — o almeno ai miei — fanno pensare alla filosofia della storia, alla religione, tutt'al più al lavoro del docente in chiave di educazione civica.

Io ho insegnato quindici anni Italiano e Storia, non è che sia insensibile ai problemi della comunicazione didattica; ma, forse perché erano ragazzi grandi dei Tecnici e delle Magistrali, non ricordo di aver dovuto particolarmente mediare, rispetto ai libri che stavo allora pensando e scrivendo, I vinti di Caporetto e Il mito della Grande guerra. Capisco che il problema si possa porre in maniera ben più stringente per un insegnante delle elementari o delle medie, come mi pare siano molti dei vostri associati.

Ora, accade questo, andando in giro come ho fatto e faccio io - quasi senza soluzione di continuità — dal 2011 a ora, in chiave di anniversari: che quasi tutti gli ascoltatori sembrano vogliosi e predisposti agli appelli ai valori — adesso, per il '14-'18, della pace soprattutto a fare buone politiche della memoria, incisiva educazione civica. Questi sono i migliori e i più motivati, naturalmente, e sono spesso insegnanti, impegnati nella trasmissione fra generazioni. Altri sono, più biecamente, dei relativisti: così è se mi pare.

Visto che io nella divulgazione credo, non mi sottraggo a questi contatti, però sono convinto di dovermi ritagliare un ruolo professionale mio proprio - da storico - di chi chiama a rispettare i fatti, il vissuto, il passato come è stato e non come si vorrebbe che fosse stato. Il 1914-1918 — nella luce edificante di cento anni dopo — sta diventando solo l' "inutile strage" del papa di allora: una frase che appena sfuggita in pubblico, le gerarchie cattoliche hanno in realtà fatto di tutto per nascondere sotto il tappeto come una... fatta di cane! E ora invece fa da buonsensaio "lieto fine" e sembra che tutti la pensassero così. Salvo quell' "assassino" di Cadorna, naturalmente (il cattolicissimo Cadorna, pieno di figlie suore e di pie donne).

Vede che non faccio al vostro caso? Lo storico può e, forse, in certi casi, deve dare scandalo. L'insegnante ha dei problemi diversi. 
Anche gli Istituti di storia della Resistenza hanno fatto e fanno politica della memoria, non è che non lo sappiamo ${ }^{18}$; e quando, a un certo punto della loro vita, gli storici si sono aggiunti ai partigiani che li avevano messi in piedi, gli equilibri fra memoria, politica della memoria, educazione civica e storiografia si sono modificati, senza mai giungere a separazioni radicali fra le sensibilità e i piani del discorso pubblico. Anche le Società di storia patria, al loro tempo, o - negli organigrammi accademici - la Storia del Risorgimento come la Storia dei partiti e dei movimenti politici o la Storia del movimento operaio sono nate (e morte) risentendo di spinte e contingenze. Il passato c'è perché il presente lo tiene in vita. E la nomenclatura viaria, i monumenti. Lo sappiamo che la lettura e la ricostruzione della storia contemporanea non avvengono in apnea. Però, fra tante urgenze e pressioni e trascendimenti virtuosi dei nudi fatti, potrebbe considerarsi interesse comune preservare qualche zona di rispetto con custode.

Sarebbe stato bello. Certo, sarebbe stato bello. Dice che le tradizioni si inventano $^{19}$. E anche le favole non sono sempre le stesse e si possono cambiare: da Cappuccetto rosso a Rodari. I neutralisti invadono le piazze e ne espellono gli interventisti. Partito socialista e Cgl proclamano in tutta Italia un vittorioso sciopero generale. Corridoni e i De Ambris si reiscrivono al partito. Riapre il Parlamento e Giolitti sostituisce Salandra.

E via fantasticando.

Non è poi solo uno scherzo. Omettendo e forzando, il "sì" alla guerra consorziato e frontista - ha depotenziato i "no". Poi l'interventismo nazionalista ha imposto le sue sovradeterminazioni all'interventismo liberaldemocratico e riformista. Il racconto sociale della Grande guerra ne ha reso unanime la narrazione nell'Italia nazionalfascista. E avanti così, ogni periodo mettendoci del suo. L'autonarrazione è avanti tutto esclusione. Perché, regnando papa Francesco, il $m c d$ della non violenza e dell'odio a tutte le guerre non dovrebbe provare a riesumare papa Benedetto XV e a proporre l'“inutile strage" come retta interpretazione degli umani e logici sentimenti di allora?

Cambiare, saper cambiare. Non sono uno specialista in materia. Ma ecco che, per una volta che prendo atto di come veramente sono andate le cose e ne modifico la visione, alt, non si può, com'era verde la tua vallata: caporettista jusqu'au bout.

Chiudo ora La guerra verticale, il libro di Diego Leoni su Uomini, animali e macchine sul fronte di montagna 1915-191820. Contende a Quinto Antonel-

\footnotetext{
${ }^{18} \mathrm{Si}$ veda: http://www.novecento.org/uso-pubblico-della-storia/intervista-a-mario-isnenghisul-centenario-della-prima-guerra-mondiale-1389/.

${ }^{19} \mathrm{Si}$ veda Eric J. Hobsbawm, Terence O. Ranger (a cura di), L'invenzione della tradizione, Torino, Einaudi, 1987.

${ }^{20} \mathrm{Si}$ veda: Diego Leoni, La guerra verticale. Uomini, animali e macchine sul fronte di montagna, 1915-1918, Torino, Einaudi, 2015.
} 
li, Storia intima della Grande guerra ${ }^{21}$ il ruolo di libro più sostanzioso nello spicchio di centenario sin qui consumato. Ma Lettere, diari e memorie dei soldati al fronte corona una indagine pluridecennale, di questo ed altri ricercatori, nel quadrato della scrittura popolare Rovereto-Trento-Pieve S. Stefano-Genova. Il primo risulta a questo punto più inventivo, anche se accompagna altri due recenti buoni libri sulle Alpi, di Marco Armiero ${ }^{22}$ e Antonio De Rossi ${ }^{23}$, concentrando l'attenzione sul tempo della guerra. Non sarà un caso che proprio due componenti del gruppo di Rovereto si presentino all'appuntamento con lavori di un tale spessore, a differenza di tanti altri d'occasione: opere che vengono da lontano. Prese le mosse di qui, dai margini, nel famoso convegno del 1985 a Rovereto ${ }^{24}$ - la periferia che si faceva centro - la liquefazione dello Stato; e anche dell'oggetto, con quel militare in copertina (perché la casa editrice Il Mulino non ha mai riedito e ha lasciato esaurito un lavoro tanto innovativo e periodizzante?) che si dissolveva in fantasma. Partiti prima - sconfinando nei luoghi del confine - verso una seriale moltitudine di "io" di massa con e senza divisa, stanno forse arrivando anche prima a forme di restituzione dell'oggetto: non più lungo confini di classe e di Stato, e neppure, a rigore, di esercito, mettendo a frutto le libertà e le coazioni della natura in micro e macro-spazi a geometria variabile.

Antonio Gibelli ${ }^{25}$, fra gli scopritori della "scrittura popolare" ed anche fra i più influenzati dalla storiografia francese, non ha comunque accettato di restare prigioniero del paradigma vittimario ed è infatti tra i pochi che si misurino con la storia complessiva della guerra. Abbiamo agito in parallelo come consulenti del ciclo delle venti puntate di Rai Storia, distribuite anche in Cd dal "Corriere della Sera". Vedo ora in Il colpo di tuono - il titolo di sapore manniano che raccoglie i suoi saggi dandogli per Manifestolibri anche un sottotitolo a chiave: Pensare la Grande guerra oggi - che Gibelli fa cominciare la nuova storiografia della Prima guerra mondiale da Fussell, Leed e dal convegno di Rovereto che li lancia nel 1985 in Italia. Aprendo il suo libro d'oggi, uno dei curatori del volume di atti di allora, Leoni, ricorda però di avere proprio lui portato nel 1985 Leed sui monti trentini, dove lo storico statunitense vagava pensoso bofonchiando fra sé di non sapere nulla di questo fronte e di questa guerra, e di

${ }^{21} \mathrm{Si}$ veda: Quinto Antonelli, Storia intima della grande guerra. Lettere, diari e memorie dei soldati dal fronte, con un Dvd del film di Enrico Verra Scemi di guerra, Roma, Donzelli, 2014.

${ }^{22}$ Si veda: Marco Armerio, Le montagne della patria. Natura e nazione nella storia d'Italia secoli XIX e XX, Torino, Einaudi, 2013.

${ }^{23}$ Si veda: Antonio De Rossi, La costruzione delle Alpi. Immagini e scenari del pittoresco alpino (1773-1914), Roma, Donzelli, 2014.

${ }^{24} \mathrm{Si}$ veda: Diego Leoni e Camillo Zadra (a cura di), La Grande guerra. Esperienza, memoria, immagini, Bologna, Il Mulino, 1986.

${ }^{25}$ Si vedano: Antonio Gibelli, Il colpo di tuono. Pensare la Grande guerra oggi, Roma, Manifestolibri, 2015; e Id., La guerra grande. Storie di gente comune 1914-1919, Roma-Bari, Laterza, 2014. 
voler riparare. Non lo farà, provvederà al suo posto Leoni. Altri, all'estero, non sono arrivati neanche alla ammissione imbarazzata di Leed e hanno continuato a ignorare il fronte italo-austriaco, accreditandosi nel contempo come autori di storie "europee" a tutto campo. Io ho sempre sbuffato davanti a questo e alle genuflessioncelle d'uso di editori e recensori italiani. Sono certamente più autarchico, o più protezionista. Gibelli, che più di altri ha cercato di mediare, vedo che in questo suo ultimo libro - certo, con più grazia - non ne può più neanche lui di fronte alla "perdurante disattenzione storiografica verso situazioni come quella italiana" (p. 224). Ed è curioso che segni di nuovo interesse vengano semmai dalla storiografia in lingua tedesca - cioè dai nemici di cento anni fa - che da quella in inglese o in francese, cioè dagli ex alleati. Permane stridente il contrasto con il precoce e, invece, generoso Scene della guerra d'Italia di uno studioso come George Macaulay Trevelyan, che la storia e il fronte italiani li aveva praticati di persona (edito nel 1919, compare fra i motivati ricuperi dell'anniversario nel $2014^{26}$ ). Oswald Überegger, lo studioso della Libera università di Bolzano, è il socio di Nicola Labanca nell'esperimento di "storia trans-nazionale" della Prima guerra mondiale, auspicato e lanciato sin dal titolo della introduzione come un necessario salto di qualità e concretizzato intanto in La guerra italo-austriaca (1915-1918) ${ }^{27}$, dove fra le presenze di maggiore spessore e originalità si notano appunto quelle austro-tedesche.

Per via del persistente squilibrio Überegger si distingue anche nella raccolta di vari autori allestita dal "Corriere della Sera": 24 maggio 1915. L'Italia è in guerra $^{28}$ (a cura di Antonio Carioti e Paolo Rastelli), descrivendo con spirito libero Il punto di vista dell'Austria-Ungheria e nominando fra gli altri lo storico tedesco Helger Afflerbach, che considera l'intervento italiano "uno degli avvenimenti politici centrali del XX secolo" (pp. 101-2). Questo libro è diverso, non frutto degli archivi ricchissimi del "Corriere della Sera" e della Fondazione omonima che li va valorizzando, anche in relazione al luogo di elaborazione e di comando che fu il quotidiano di Luigi e Alberto Albertini nel 1914-1918. Vien da chiedersi quanto di più sapremmo sui ceti dirigenti e le interne tensioni della società italiana in guerra, se anche altri grandi quotidiani borghesi avessero precostituito e reso pubblici materiali d'archivio commensurabili: ad esempio, per l'Italia settentrionale, centrale e meridionale, tre testate del peso e della rappresentatività di "La Stampa" giolittiana e neutralista a Torino, e per i triplicisti e germoanofili "Il Mattino" a Napoli, o "La Nazione" di Firenze. Di che complicare e dialettizzare il quadro, rispetto all'interventismo, che esce in-

\footnotetext{
${ }^{26}$ Si veda; George Macaulay Trevelyan, Scene della guerra d'Italia, introduzione di Mario Isnenghi, Roma, Edizioni di storia e letteratura, 2014.

${ }^{27} \mathrm{Si}$ vedano: Nicola Labanca e Oswald Überegger (a cura di), La guerra italo-austriaca 1915-18, Bologna, Il Mulino, 2014; Nicola Labanca, The Italian Front, in Jay Winter (a cura di), The Cambridge History of the First Wolrd War, vol. I, Global War, Cambridge, Cambridge University Press, 2014.

${ }^{28}$ Si veda: 24 maggio 1915 l'Italia è in guerra, Milano, Corriere della Sera, 2015.
} 
vece rinforzato da questa sproporzione nel senso di sé e della propria storia da parte dei maggiori quotidiani, di allora e di oggi. Pur allargando il campo della ricerca ai socialisti, anche la benemerita raccolta di studi territoriali sul neutralismo promossa e pubblicata da Fulvio Cammarano alla testa di numerosi ricercator $\mathrm{i}^{29}$ offre in maniera sistematica tutta una serie di scavi locali, ma neppur essa sposta gli accenti rispetto all'egemonia di fatto esercitata comunque dal precario amalgama dei fautori dell'intervento.

${ }^{29} \mathrm{Si}$ veda: Fulvio Cammarano (a cura di), Abbasso la guerra! Neutralisti in piazza alla vigilia della Prima guerra mondiale in Italia, Firenze, Le Monnier, 2015. 